\title{
JoInt Signaling TeChNiQUeS FOR CROSSTALK-DOMINATED COMMUNICATION CHANNELS
}

\author{
Rohit V. Gaikwad and Richard G. Baraniuk \\ Department of Electrical and Computer Engineering \\ 6100 Main Street, Rice University \\ Houston, Texas 77005
}

\begin{abstract}
We present a framework for maximizing the capacity (or bit rate) of symmetric bit-rate communication services dominated by crosstalk, in particular Digital Subscriber Line (DSL) services. We assume the DSL channel is a Gaussian channel, so transmit power spectral density (PSD) design is sufficient to maximize bit rates. Using the channel, noise, and crosstalk transfer functions, we set up an optimization problem and solve for transmit PSDs that maximize the joint capacity of same-service users. Joint signaling techniques and optimal power distribution yield significant gains in bit rates (or performance margins) over current schemes. Furthermore, the designed transmit PSDs are inherently spectrally compatible with existing services on neighboring lines. We purposefully design a general framework; it does not depend on the exact choice of modulation scheme, for example. It is also extremely simple and of low computational complexity. The framework can also apply to other channels besides DSLs, such as wireless channels, coaxial cables, power lines, and telemetry cables used in geophysical well-logging tools.
\end{abstract}

Keywords-Digital Subscriber Line services, crosstalk, information rates, multiuser communication, spectral compatibility.

\section{Introduction}

Many communication systems rely on significant resource-sharing among multiple users, in particular transmission bandwidth. Proximity of different paths or channels between users can lead to multiuser interference or crosstalk, as in crosstalk between digital subscriber lines (DSLs) in a telephone cable or between users in a wireless channel. Crosstalk lowers channel capacities and can be a limiting factor for achievable bit rates [1]. Complete cancellation or suppression of crosstalk is not always possible, since it is difficult and/or expensive [2]. Rather, we employ crosstalk avoidance techniques and optimal spectral allocation schemes among multiple users to maximize bit rates.

For concreteness, we frame our discussion in terms of the DSL technology, though our results have more general applicability. We define a service as a transmission protocol (based on bit-rate and transmit power characteristics) over a standard telephone line. A service can be a DSL service, as, ADSL, VDSL, or HDSL [3], or a non-DSL service, as, T1, ISDN, or POTS [3]. A telephone cable in the USA typically contains up to 50 lines packed closely together, each potentially carrying a different service. Every

This work was supported by the National Science Foundation, grant no. MIP-9457438, Nortel Networks, and SBC Communications. Email: \{rohitg, richb\} @ rice.edu Web: www.dsp.rice.edu

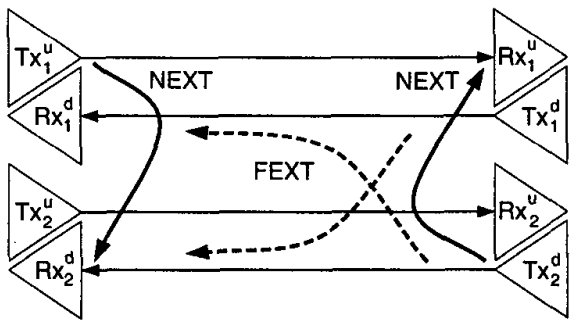

Fig. 1. Near-end crosstalk (NEXT) and far-end crosstalk (FEXT) between neighboring lines in a binder. Tx's are transmitters and $R x$ 's are receivers. A 4-2 line hybrid circuit connects the transceivers $\left(\mathrm{Tx}_{1}^{u} / \mathrm{Rx}_{1}^{u}\right.$ pair, for example) to the telephone lines.

transmitter intends to communicate with the corresponding receiver at the other end. However, transmitted energy from a given line leaks, via electro-magnetic coupling, into other receivers of neighboring lines as a result of the proximity of lines and the absence of proper electro-magnetic shielding. This leakage leads to significant crosstalk between lines in a telephone cable (see Figure 1). As more and more DSL services are introduced, it leads to increased congestion in frequency and contention for bandwidth, for example, VDSL and Home phoneline transceivers [4].

Assuming that the DSL channel is a Gaussian channel, second-order statistics are sufficient to characterize the channel [5]. Hence, design of optimal transmit power spectral densities (PSDs), also referred to as transmit spectra, are sufficient to maximize the bit rates. Further, when we optimize the bit rate of any service we also need to make sure that the optimized service does not significantly inject crosstalk power into the existing DSL services. In other words, we must ensure that the service being optimized is spectrally compatible with existing services. A similar problem was first solved in [6] for symmetric-bit-rate services facing only self-NEXT and white AGN. Here, we solve the problem for symmetric-bit-rate [7] services in the presence of self-NEXT, self-FEXT, AGN, and interference from other services. Given the channel, noise, and crosstalk characteristics we solve an optimization problem to maximize the juint channel capacity of same-service neighboring lines under an average power (see $[8,9]$ for details) and a combined average plus peak frequency-domain power con- 
straint (details in this paper). Constrained optimal spectra yield large performance margin gains up to $13 \mathrm{~dB}$ over current fixed-spectra schemes (see Table IV-B). Further, they are inherently spectrally compatible with existing services. Constrained optimal spectra can be implemented using any modulation scheme and can be easily adapted to varying noise and interference environments.

Section II outlines our definitions, notation, and assumptions. Section III presents algorithms to obtain optimal transmit spectra. We discuss simulation results in Section IV and present conclusions in Section V.

\section{Definitions, notation, and assumptions}

There are two types of crosstalk (see Figure 1):

NEXT: Interference between neighboring lines that arises when signals are transmitted in opposite directions. If the neighboring lines carry the same type of service, then the interference is called self-NEXT.

FEXT: Interference between neighboring lines that arises when signals are transmitted in the same direction. If the neighboring lines carry the same type of service, then the interference is called self-FEXT.

The term self-interference refers to the total combined self-NEXT and self-FEXT.

We assume that the channel can be characterized as a linear time-invariant system. We divide the transmission bandwidth $B$ of the channel into $K$ narrow frequency bins; each of width $W \mathrm{~Hz}$ and center frequencies $f_{k}$ with index $k \in\{1, \ldots, K\}$. We assume that the channel, noise, and the crosstalk characteristics vary slowly enough with frequency that they can be approximated as constant over each bin [10]. We use the following notation for the magnitudesquared channel transfer function [11],

$$
H_{k}=\left|H_{C}\left(f_{k}\right)\right|^{2}, \text { for }\left|f-f_{k}\right| \leq \frac{W}{2}, 0 \leq f \leq B
$$

self-NEXT transfer function [12],

$$
X_{k}=\left|H_{N}\left(f_{k}\right)\right|^{2}, \text { for }\left|f-f_{k}\right| \leq \frac{W}{2}, 0 \leq f \leq B
$$

and self-FEXT transfer function [12]

$$
F_{k}=\left|H_{F}\left(f_{k}\right)\right|^{2}, \text { for }\left|f-f_{k}\right| \leq \frac{W}{2}, 0 \leq f \leq B .
$$

We consider real signals with symmetric frequency responses. Thus, we deal only with quantities over the nonnegative frequency region.

In addition we assume that:

1. The channel transfer function is monotone decreasing. In case the channel transfer function is non-monotonic (e.g., in the case of bridged taps on the line [3]), the techniques that follow can be applied in each individual bin independently. This scenario makes the power distribution problem more difficult, however (see [9] for details).

2. The level of self-FEXT is small enough in all the bins that it is not necessary to separate in frequency samedirection transmit spectra of same-service lines.

3. Channel noise $\left(N_{o}(f)\right)$ can be modeled as AGN. We assume that we have no control over the transmit spectra of existing services and treat this different-service NEXT $\left(D S_{N}(f)\right)$ and $\operatorname{FEXT}\left(\left(D S_{N}(f)\right)\right.$ as AGN [13] for capacity purposes. We combine the $D S_{N}(f), D S_{N}(f)$, and channel noise to obtain the total Gaussian noise [13] as

$$
N_{k}:=N\left(f_{k}\right):=N_{o}\left(f_{k}\right)+D S_{N}\left(f_{k}\right)+D S_{F}\left(f_{k}\right) \text {. }
$$

4. Symmetric bit-rates are used in both directions of transmission for all the same-service lines. Further, we assume that the channel, self-NEXT, self-FEXT, different-service interference, and channel noise are the same for all sameservice lines. Symmetric bit-rates implies that all the upstream PSDs $\left(S^{u}(f)\right)$ are the same and all the downstream PSDs $\left(S^{d}(f)\right)$ are the same of the same-service lines.

DSL modems transmit in two directions on the same line via a 4-2 line hybrid circuit. A No Division Signaling (NDS) ${ }^{1}$ scheme in frequency bin $k$ is one for which $S^{u}(f)=S^{d}(f) \neq 0$ for all $f$ in the bin. (that is, both upstream and downstream transmissions occupy the band $\left|f-f_{k}\right| \leq \frac{W}{2}$ in the same way). A Frequency Division Signaling (FDS) scheme in frequency bin $k$ is one for which $S^{u}(f)=0$ when $S^{d}(f) \neq 0$ for all $f$ in the bin and vice versa (that is, both transmissions occupy orthogonal frequency bands within $\left.\left|f-f_{k}\right| \leq \frac{W}{2}\right)$. FDS is an example of the general concept of orthogonal signaling that includes other schemes like time-division signaling (TDS) and codedivision signaling (CDS). FDS eliminates self-NEXT and therefore potentially increases capacity; however, FDS also cuts the transmission bandwidth in half thus potentially reducing capacity. Clearly, NDS/FDS should be employed judiciously to optimize this tradeoff.

\section{Optimized signaling techniques}

\section{A. Optimization under an average power constraint}

Problem statement: Maximize the capacity of a DSL service in the presence of channel noise, interference (DSNEXT and DS-FEXT) from other services, and self-NEXT and self-FEXT under two constraints: (1) the average DSL input power in each direction of transmission be limited to $P_{\max }$, and (2) equal capacity in both directions (upstream and downstream) of transmission.

\footnotetext{
${ }^{1}$ NDS was referred to as EQPSD signaling in $[6,8]$ and our standards contributions $[7,14,15]$
} 
In the absence of self-interference, the interference combination consists exclusively of different service interferers and AGN. This total interference can be lumped together into one AGN source as in (4) [13]. Under the Gaussian channel assumption the optimal power distribution in each direction of transmission is obtained by the classical waterfilling solution [16].

When present, self-NEXT and self-FEXT severely limit the achievable bit rates in symmetric-bit-rate DSL services. In this paper (and for long lines with small bandwidths in general) self-NEXT can be assumed to dominate self-FEXT and self-FEXT can be assumed small [9]. This is the case of interest for high-bit-rate DSL2 (HDSL2) [12]. However, self-FEXT still factors into our design in a significant way. This is a new, non-trivial extension of the work of [6].

\section{A.1 Joint signaling scheme}

The level of self-NEXT varies with frequency. In high self-NEXT regions FDS might be useful to reject the selfNEXT. However, in low self-NEXT regions, the loss of transmission bandwidth of FDS could outweigh any capacity gain due to self-NEXT rejection. Therefore, we would like our signaling scheme to be general enough to encompass both FDS, NDS, and the array of choices in between. Our approach is related to that of [6].

Consider the case of two neighboring lines carrying the same service. Line 1 upstream capacity is $C^{u}$ and line 2 downstream capacity is $C^{d}$. Under the Gaussian channel assumption, we can write the upstream capacity in bits per second (bps) as

$$
\begin{aligned}
& C^{u}=\sup _{S^{u}(f), S^{d}(f)} \int_{0}^{\infty} \log _{2}[1+ \\
& \left.\frac{\left|H_{C}(f)\right|^{2} S^{u}(f)}{N(f)+\left|H_{N}(f)\right|^{2} S^{d}(f)+\left|H_{F}(f)\right|^{2} S^{u}(f)}\right] d f .
\end{aligned}
$$

The supremum is taken over all possible $S^{u}(f)$ and $S^{d}(f)$ satisfying

$$
S^{u}(f) \geq 0, \quad S^{d}(f) \geq 0 \quad \forall f,
$$

and the average power constraints for the two directions

$$
2 \int_{0}^{\infty} S^{u}(f) d f \leq P_{\max }, \quad 2 \int_{0}^{\infty} S^{d}(f) d f \leq P_{\max } .
$$

$C^{d}$ can be expressed in a similar manner by interchanging $S^{u}(f)$ and $S^{d}(f)$ in the above equations for $C^{u}$. We can solve for the capacities $C^{u}$ and $C^{d}$ using classical waterfilling [16] if we impose the restriction of NDS, that is $S^{u}(f)=S^{d}(f) \forall f$. However, this gives low capacities. Therefore, we employ FDS $\left(S^{u}(f)\right.$ orthogonal to $\left.S^{d}(f)\right)$ in spectral regions where self-NEXT is large enough to limit our capacity and NDS in the remaining spectrum. This gives much improved performance $[8,9]$.

\section{A.2 Optimal signaling scheme: One frequency bin}

We continue our analysis on a single frequency bin $k$ assuming the frequency bin responses (1)-(3). Using standard optimization techniques we can solve for the correct signaling scheme that maximizes capacities in each bin (see $[8,9]$ for details). We can write test conditions to determine the signaling nature (NDS or FDS) in a given bin:

$$
\begin{aligned}
& \text { if } X_{k}^{2}-F_{k}^{2}-H_{k} F_{k}<0 \text {, then } \\
& G_{k}=\frac{2 P_{m}}{N_{k} W} \underset{\mathrm{FDS}}{\mathrm{NDS}} \frac{H_{k}-2\left(X_{k}-F_{k}\right)}{X_{k}^{2}-F_{k}^{2}-H_{k} F_{k}} \\
& \text { else if } X_{k}^{2}-F_{K}^{2}-H_{k} F_{k}>0 \text {, then }
\end{aligned}
$$

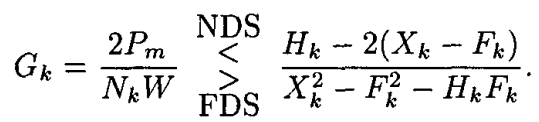

The test conditions imply perform FDS in the bin if the SNR $\left(G_{k}\right)$ is low as compared to the self-interference $\left(X_{k}, F_{k}\right)$ or NDS otherwise.

Please note that FDS is a special case of the more general orthogonal signaling concept, for example, TDS and CDS. However, of all orthogonal signaling schemes, FDS signaling gives the best results in terms of spectral compatibility under an average power constraint and hence is used here (see proof in [9]).

\section{A.3 Optimal spectra: All frequency bins}

To obtain the signaling schemes for all bins, we apply the test conditions (8) and (9) to each frequency bin in $[0, B]$. In the low self-FEXT case we obtain an NDS frequency region to the left of a switch-over bin $M_{\mathrm{N} 2 \mathrm{~F}}$ and FDS frequency region to the right of it [9]. We perform optimal power distribution over all bins according to the algorithm in [10]. A simple iterative algorithm yields the complete optimal transmit spectra (see [8] and [9] for more details).

We can reduce the iterative algorithm to a single, computationally efficient step and obtain a fast near-optimal solution.

\section{B. Optimization under a frequency-domain peak power constraint: No self-interference}

A peak power constraint in the frequency-domain could arise in a number of ways: (1) peak power limit, (2) a standardized PSD mask, or (3) from spectral compatibility considerations. In this section we will impose an additional frequency-domain peak power constraint. This constraint is in addition to the average power constraint. We obtain optimal transmit spectra for a DSL service under these constraints, in the absence of self-interference. 
The problem statement is similar to that in Section IIIA but without the equal capacity constraint and with additional power constraints. Consider a line carrying a DSL service in the presence of different-service interference and channel noise. The twisted pair channel can be treated as a Gaussian channel with colored Gaussian noise [13]. The channel capacity (in bps) is given by (5) [16] with the average power constraints (6) and (7). In addition we have new peak power constraints

$$
S^{u}(f) \leq Q^{u}(f) \forall f \quad \text { and } \quad S^{d}(f) \leq Q^{d}(f) \forall f .
$$

For discussion purposes, we will focus on the upstream transmission. The same analysis can be applied to the downstream channel.

We wish to maximize (5) subject to the constraints (6), (7), and (10). The constraints are linear and differentiable. Further, the objective function to be maximized (5) is concave (the $\log$ function is concave). Any solution to this problem must satisfy the Karush-Kuhn-Tucker (KKT) [17] necessary conditions for optimality. For a concave objective function and linear, differentiable constraints, any solution that satisfies the necessary KKT conditions is a unique globally optimal solution [17]. Thus, we seek any solution that satisfies the necessary KKT conditions.

"Peak-constrained water-filling"2 yields the optimal solution to (5), (6), (7), and (10). The optimal transmit spectrum is given by

$$
S_{\mathrm{opt}}^{u}(f)= \begin{cases}\lambda-\frac{\left.N_{\mathrm{o}}(f)\right)+D S_{N}(f)+D S_{F}(f)}{\left|H_{C}(f)\right|^{2}} & \text { for } f \in E_{\mathrm{pos}} \\ Q^{u}(f) & \text { for } f \in E_{\max }, \\ 0 & \text { otherwise, }\end{cases}
$$

with $\lambda$ a Lagrange multiplier. The spectral regions $E_{\mathrm{pos}}$ and $E_{\max }$ are specified by

$$
\begin{aligned}
E_{\mathrm{pos}} & =\left\{f: 0 \leq S^{u}(f) \leq Q^{u}(f)\right\}, \text { and } \\
E_{\max } & =\left\{f: S^{u}(f)>Q^{u}(f)\right\} .
\end{aligned}
$$

We vary the value of $\lambda$ to achieve the optimal transmit spectrum $S_{\text {opt }}^{u}(f)$ that satisfies the average and peak power constraints (6), (7), and (10). It can be easily shown that this solution satisfies the KKT conditions for optimality. Substituting the optimal PSD $S_{\mathrm{opt}}^{u}(f)$ into (5) yields the capacity $C^{u}$ under the average and peak power constraints.

Note that if the maximum allowed average power $\left(P_{\max }\right)$ exceeds the power under the peak power constraint then the optimal transmit spectrum is the constraining PSD itself. In the absence of an average power constraint (but with a peak power constraint) the optimal transmit spectrum is again the constraining PSD.

${ }^{2}$ Peak-constrained water-filling can be likened to filling water in a closed vessel with uneven top and bottom surfaces.

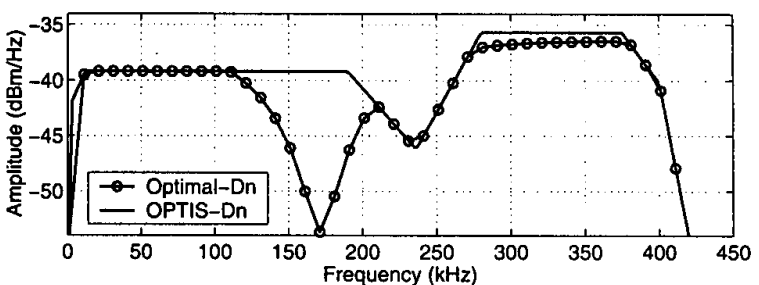

Fig. 2. Constrained downstream optimal transmit spectra for HDSL2 on CSA loop 6 with a downstream OPTIS constraining PSD mask with $49 \mathrm{HDSL}$ NEXT interferers and AGN of $-140 \mathrm{dBm} / \mathrm{Hz}$.

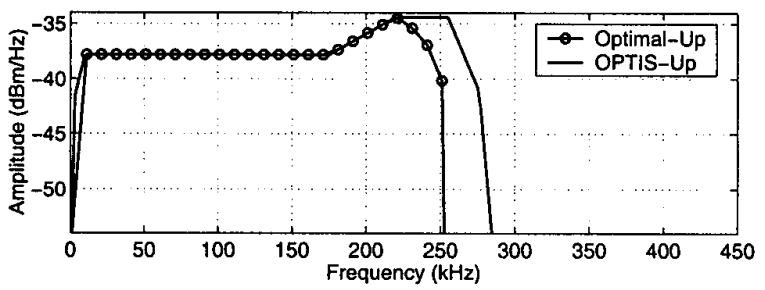

Fig. 3. Constrained upstream optimal transmit spectra for HDSL2 on CSA loop 6 with a upstream OPTIS constraining PSD mask with 25 T1 NEXT interferers and $A G N$ of $-140 \mathrm{dBm} / \mathrm{Hz}$.

\section{Simulation results and discussion}

\section{A. Examples}

Figures 2 and 3 illustrate the constrained optimal transmit spectra under the OPTIS (ANSI T1E1.4's HDSL2 standard for transmit PSD masks) PSD masks [18] carrier serving area (CSA) [3] loop 6 for HDSL2 in the presence of 49 HDSL NEXT and 25 T1 NEXT, interferers, respectively. ${ }^{3}$

In both figures, the constrained optimal spectra employ NDS signaling throughout the frequency band. Further, the constrained spectra do not necessarily follow the constraining PSD. For more examples please refer [9].

\section{B. Performance margins}

The performance margin or noise margin of a channel for a fixed bit rate and bit error rate measures the maximum degradation (from noise and interference) in performance that a channel can sustain before being unable to transmit at that bit rate and bit error rate [21].

\footnotetext{
${ }^{3}$ Simulation Details: Bit rate fixed at $1.552 \mathrm{Mbps}$. Total average input power in each direction $P_{\max }=19.78 \mathrm{dBm}$. Different service interference models obtained from Annex B of T1.413-1995 (from [11], the ADSL standard), with exceptions as in [19]. Self-NEXT interference modeled as a 2-piece Unger model [12]. Margins calculated according to [20]. OPTIS (ANSI TIE1.4's HDSL2 standard for transmit PSD masks) transmi spectra obtained by tracking $1 \mathrm{dBm} / \mathrm{Hz}$ below the OPTIS PSD masks [18] OPTIS performance margin figures from [18]. AGN of $-140 \mathrm{dBm} / \mathrm{Hz}$ added to the interference. DMT modulation scheme: Sampling frequency $f_{s}=1000 \mathrm{kHz}$. Bin width $W=2 \mathrm{kHz}$. Number of bins $K=250$. Start frequency $=1 \mathrm{kHz}$. Bit error rate $=10^{-7}$. SNR gap $=9.8 \mathrm{~dB}$. No cyclic prefix. No limitation on maximum number of bits per tone. See [21] for more details.
} 
Table IV-B compares the uncoded performance margins ${ }^{3}$ of the constrained optimal transmit spectra "under OPTIS" versus the OPTIS transmit spectra [18] for CSA loop 6. For different service interferers (HDSL and T1), only the NEXT powers were considered, while for HDSL2 "self" comprises both self-NEXT and self-FEXT. Constrained-optimal transmit spectra always outperform (have higher performance margins) fixed, standard (non-adaptive) spectra. The margins of constrained optimal spectra are smaller than ones obtained using optimal spectra [8] because of the constraining PSD masks.

Table I: Uncoded (no coding gains) performance margins (in $d B$ ) for HDSL2 on CSA Ioop 6: OPTIS vs. constrained optimal.

\begin{tabular}{|l|c|c|c|c|c|c|}
\hline \multirow{2}{*}{ Crosstalk } & \multicolumn{2}{|c|}{ OPTIS } & \multicolumn{2}{c|}{ Optimal } & \multicolumn{2}{c|}{ Diff } \\
\cline { 2 - 7 } & Up & Dn & Up & Dn & Up & Dn \\
\hline \hline 49 HDSL & 2.7 & 12.2 & 3.7 & 13.8 & $\mathbf{1 . 0}$ & $\mathbf{1 . 6}$ \\
\hline 25 T1 & 19.9 & 17.5 & 20.4 & 18.8 & $\mathbf{0 . 5}$ & $\mathbf{1 . 3}$ \\
\hline 39 self & 2.1 & 9.0 & 15.5 & 17.6 & $\mathbf{1 3 . 4}$ & $\mathbf{8 . 6}$ \\
\hline 24 self +24 T1 & 4.3 & 1.7 & 4.5 & 4.7 & $\mathbf{0 . 2}$ & $\mathbf{3 . 0}$ \\
\hline
\end{tabular}

\section{Conclusions}

In this paper, we have derived optimal transmit spectra for symmetric bit-rate communication channels dominated by self-NEXT, in particular for DSLs. We setup and solved an optimization problem to jointly maximize the bit rates of each same-service DSL line in a binder given the channel, noise, and crosstalk characteristics. Constrained optimal spectra vary with interference combination and do not necessarily follow the constraining PSD.

The key advantages of our techniques are: (1) Optimal transmit spectra yield large performance margin gains (up to $13 \mathrm{~dB}$ in our experience) as compared to current fixedspectra schemes. We can trade these increased performance margins for increased bit rates or decreased average transmission power (which in turn will reduce crosstalk). (2) Optimal transmit spectra are inherently spectrally compatible with existing services. (3) Optimal spectra are not bound to any particular modulation scheme. (4) Transmit spectra can be adapted on-line to changes in line conditions (e.g., temperature variations, etc.). (5) Optimal spectra can be used for spectrum management in a telephone cable. (6) Vendor advantage can be achieved by using adaptive constrained optimal spectra underneath fixed, standardized spectra.

For details on obtaining optimal spectra under only an average power constraint and in the presence of bridged taps please refer to $[8,9]$.

Our scheme requires a priori knowledge of the characteristics of the neighboring interfering services. In a practical system, these can either be estimated at start-up or analyzed in a worst-case manner for a particular line under consideration. This information could also be obtained from a central office database that specifies the type of services in each binder group in the telephone cable.

\section{References}

[1] I. Kalet, "The Multitone Channel," IEEE Trans. Commun., vol. 37, Feb. 1989.

[2] S. Verdu, Multiuser Detection. Cambridge University Press, 1998.

[3] W. Chen, DSL Simulation Techniques and Standards Development for Digital Subscriber Line Systems. Macmillan Technology Series, 1998.

[4] D. Shaver, R. Gaikwad, and et.al., "G.PNT: Spectral compatibility of home phoneline network transeivers (g.pnt) with xdsl and other existing in-home services," ITU-T Q4/SG15 Contribution, no. NG102, 1999.

[5] J. Werner, "The HDSL Environment," IEEE J. Select. Areas Commun., vol. 9, pp. 785-800, Aug. 1991.

[6] A. Sendonaris, V. Veeravalli, and B. Aazhang, "Joint Signaling Strategies for Approaching the Capacity of Twisted Pair Channels," IEEE Trans. Commun., vol. 46, May 1998.

[7] R. Gaikwad and R. Baraniuk, "Optimal Transmit Spectra for HDSL2," ANSI TIEl Contribution, no. T1E1.4/98-162R1, 1998.

[8] R. Gaikwad and R. Baraniuk, "Optimal Transmit Spectra for Communication in the Presence of Crosstalk," ICC, June 1999.

[9] R. Gaikwad and R. Baraniuk, "Spectral optimization and joint signaling techniques for communication in the presence of crosstalk," Tech. Rep. 9806, Rice University, Electrical and Computer Engineering Department, Houston, Texas, July 1998. Web: www.dsp.rice.edu/publications/Papers/tr9806.ps.

[10] J. Aslanis and J. Cioffi, "Achievable Information Rates on Digital Subscriber Loops: Limiting Information Rates with Crosstalk Noise," ANSI TIEI Contribution, vol. 40, Feb. 1992.

[11] American National Standard for Telecommunications, "Network and Customer Installation Interfaces- Asymmetric Digital Subscriber Line (ADSL) Metallic Interface," ANSI TIEI Standard, no. T1.413. $1995,1995$.

[12] K. Kerpez, "Full-duplex 2B1Q Single-pair HDSL Performance and Spectral Compatibility," ANSI TIEI Contribution, no. T1E1.4/95127, 1995.

[13] K. Kerpez, "Near-End Crosstalk is almost Gaussian," IEEE Trans. Commun., vol. 41, Jan. 1993.

[14] R. Gaikwad and R. Baraniuk, "Optimal Transmit Spectra for HDSL2 under a Peak Frequency-Domain Power Constraint," ANSI TIEI Contribution, no. T1E1.4/98-188R2, 1998.

[15] R. Gaikwad and R. Baraniuk, "Transmit Spectra Adaptive to Noise and Interference at Activation for HDSL2," ANSI TIEI Contribution. no. T1E1.4/98-258, 1998.

[16] R. Gallager, Information Theory and Reliable Communication. New York: Wiley, 1968.

[17] R. Horst, P. Pardalos, and N. Thoai, Introduction to Global Optimiza tion. Kluwer Academic Publishers, 1995.

[18] J. Girardeau, M. Rude, H. Takatori, and G. Zimmerman, "Updated OPTIS PSD Mask and Power Specification for HDSL2," ANSI TIEI Contribution, no. T1E1.4/97-435, 1997.

[19] G. Zimmerman, "Performance and Spectral Compatibility of OPTIS HDSL2," ANSI TIEl Contribution, no. T1E1.4/97-237, 1997.

[20] G. Zimmerman, "Normative Text for Spectral Compatibility Evaluations," ANSI TIEl Contribution, no. T1E1.4/97-180R1, 1997.

[21] M. Barton and M. Honig, "Optimization of Discrete Multitone to Maintain Spectrum Compatibility with Other Transmission Systems on Twisted Copper Pairs," IEEE J. Select. Areas Commun., vol. 13, pp. 1558-1563, Dec. 1995. 